\title{
BioChangeMeadows - German meadows in the 1950s, 1990s and in 2008
}

\author{
Karsten Wesche \& Benjamin Krause
}

\begin{abstract}
The BioChangeMeadows database (GIVD ID EU-DE-008) contains samples of moist and mesic meadows in northern and central Germany, and covers both historical (1950s and 1960s) and more recent (1990s, 2008) relevés. Analyses showed that plot-level diversity has decreased tremendously over time, which was largely irrespective of landscape context. Plant community composition shifted as well with decreasing numbers of insect-pollinated herbs and increasing numbers of grasses. Functional diversity showed similarly large declines as taxomomic diversity. Historical and current vegetation maps are also available and can be retrieved from the authors upon request.
\end{abstract}

Keywords: central Europe; diversity; historical comparison; map; phytosociology.

\section{BioChangeMeadows}

Scope: Data base covers a number of case studies from moist meadow vegetation in northern Germany. The data are part of an ongoing research project hosted at the University of Göttingen (BioChange-Germany) where historical vegetation samples (and vegetation maps) from the 1950 s/60s are compared to more recent surveys and the current condition.

Both historical and present vegetation maps are available on request (ArcView format)

Status: completed and continuing

Period: 1939-2008

Database manager(s): Karsten Wesche (karsten.wesche@senckenberg.de), Benjamin Krause (bkrause@gwdg.de)

Owner: [NA]

Web address: [NA]

Availability: free upon request

Online upload: no

Online search: no

Database format(s): TURBOVEG

Export format(s): [NA]

Publication: Wesche, K., Krause, B., Culmsee, H. \& Leuschner, C. 2009. Veränderungen in der Flächen-Ausdehnung und

Artenzusammensetzung des Feuchtgrünlandes in Norddeutschland seit den 1950er Jahren. Berichte der Reinhold-Tüxen-Gesellschaft 21: 196210.

Plot type(s): normal plots

Non-overlapping plots: 1,092

Total plot observations: 1,092

Plot-size range: $15-25 \mathrm{~m}^{2}$

Countries: DE: $100.0 \%$

Forest: [NA] — Non-forest: [NA]

Guilds: all vascular plants: $100 \%$

Environmental data: altitude: $100 \%$; slope aspect: $20 \%$; slope inclination: $20 \%$

Performance measure(s): cover: $100 \%$

Geographic localisation: GPS coordinates (precision $25 \mathrm{~m}$ or less): $60 \%$; political units or only on a coarser scale (>10 km): $40 \%$

Sampling periods: 1930-1939: 2.0\%; 1940-1949: 3.0\%; 1950-1959: 30.0\%; 1960-1969: 25.0\%; 2000-2009: 40.0\% Information as of 2012-07-12; further details and future updates available from http://www.givd.info/ID/EU-DE-009

Karsten Wesche* (karsten.wesche@senckenberg.de)

Deptartment of Botany, Senckenberg Museum of Natural History, Am Museum 1, 02826 Görlitz, GERMANY

Benjamin Krause

Abteilung Naturschutz und Landschaftspflege, Georg August Universität Göttingen, Büsgenweg 3, 37077 Göttingen, GERMANY

*Corresponding author 